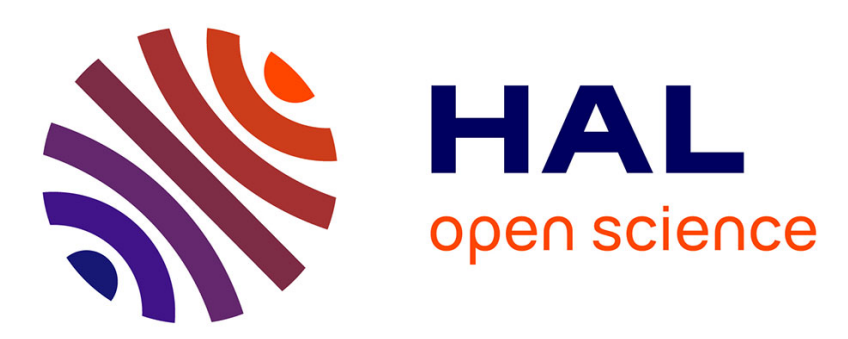

\title{
Capacity and Energy-Consumption Optimization for the Cluster-Tree Topology in IEEE 802.15.4
}

Fabrice Theoleyre, Benoit Darties

\section{To cite this version:}

Fabrice Theoleyre, Benoit Darties. Capacity and Energy-Consumption Optimization for the ClusterTree Topology in IEEE 802.15.4. IEEE Communications Letters, 2011, 15 (8), pp.816-818. hal00923365

\section{HAL Id: hal-00923365 \\ https://hal.science/hal-00923365}

Submitted on 2 Jan 2014

HAL is a multi-disciplinary open access archive for the deposit and dissemination of scientific research documents, whether they are published or not. The documents may come from teaching and research institutions in France or abroad, or from public or private research centers.
L'archive ouverte pluridisciplinaire HAL, est destinée au dépôt et à la diffusion de documents scientifiques de niveau recherche, publiés ou non, émanant des établissements d'enseignement et de recherche français ou étrangers, des laboratoires publics ou privés. 


\title{
Capacity and Energy-Consumption Optimization for the Cluster-Tree Topology in IEEE 802.15.4
}

\author{
Fabrice Theoleyre, Member, IEEE, Benoît Darties
}

\begin{abstract}
IEEE 802.15.4 proposes to use a cluster-tree hierarchy to organize the transmissions in Wireless Sensor Networks. In this letter, we propose a framework to analyze formally the capacity and the energy consumption of this structure. We derive a Mixed Integer Linear Programming (MILP) formulation to obtain a topology compliant with the standard. This formulation provides the optimal solution for the network capacity: this constitutes an upper bound for any distributed algorithms permitting to construct a cluster-tree. This framework can also be used to evaluate the capacity and to compare quantitatively different cluster-tree algorithms.
\end{abstract}

Index Terms-IEEE 802.15.4, cluster-tree, capacity, MILP

\section{INTRODUCTION \& MOTIVATIONS}

IEEE 802.15.4 [1] is a standard to inter-connect low-power sensors and actuators. It can use either the beacon-enabled mode (a coordinator sends periodical beacons) or the nonbeacon mode. To save energy in the former mode, the designers introduced the concept of superframes: a coordinator sends periodical beacons. When the superframe is terminated, all the nodes can sleep safely until the new beacon. Thus, we will here focus on the beacon-enabled mode of IEEE 802.15.4.

In multihop, IEEE 802.15.4 maintains a cluster-tree: the nodes form a tree, rooted at the PAN coordinator. All the nonleaf nodes periodically transmit a beacon to maintain their own superframe. Transmissions are then regulated via a slotted Carrier-Sense Multiple Access with Collision Avoidance (CSMA/CA). Besides, a node can exchange packets only with its parent and children in the cluster-tree.

The cluster-tree is constructed iteratively: each node first associates to one parent and then starts transmitting its own beacons. Two methods can be implemented to discover new parents: in passive scan, a node listens to the medium, waiting for a beacon. In active scan, a node triggers an explicit discovery by transmitting a solicitation: an associated node has to respond with a beacon. The former mode is more relevant for beacon-enabled IEEE 802.15.4 while the latter one is required if no beacon is periodically transmitted.

However, the standard does not specify which parent to choose (just how it associates to). Surprisingly, only few articles investigated the impact of this choice on the clustertree topology. Cuomo et al. studied in [2] the average number of children and the height of the tree while Claudios et al. investigated in [3] the dynamic properties of the structure.

The contribution of this letter is twofold:

1) we propose here an analytical framework to evaluate the throughput and the energy consumption using the radio

Manuscript received March 10, 2011

Fabrice Theoleyre is with the CNRS, University of Strasbourg, France and Benoît Darties is with the University of Burgundy, France (email: theoleyre@unistra.fr, benoit.darties@u-bourgogne.fr) topology and the conflict graph This evaluation permits to compare different cluster-tree algorithms;

2) this formulation defines the optimal cluster-tree topology concerning these criteria, providing an upper bound. This formulation could possibly be used directly if the Wireless Sensor Network (WSN) topology is pre-defined and static.

\section{MODEL \& ASSUMPTIONS}

To be as general as possible, we consider the radio bandwidth is equal to 1 unit. We assume the beacon-only period [4] is implemented: mini-slots are reserved for beacons, and the algorithm assigns disjoint mini-slots for two interfering coordinators. Thus, we assume here that beacons do not collide, i.e. no superframe is lost because of beacon's collisions.

CSMA-CA slotted mode does not permit to avoid entirely collisions inside a superframe when the medium has to forward a large number of data packets. In particular Pollin et al. demonstrated in [5] that the percentage of radio bandwidth wasted by collisions is directly linked with the number of contenders. To estimate collisions, we interpolate [5], section V, fig. 9. Bandwidth waste due to collisions $B W_{\text {coll }}$ can be modeled as proportional to the number of contenders:

$$
B W_{\text {coll }}=n b \_ \text {stations } * 0.018675+0.0104
$$

with the coefficient of determination 0.97 . Thus, at most 53 stations should contend in a superframe, else IEEE 802.15.4 is inefficient to solve collision and the bandwidth is almost null.

According to IEEE 802.15.4, the superframe of a node directly follows the superframe of its parent. If we assume that all the nodes have the same $B I$ (period of beacons) and $S D$ (superframe duration) values and if we neglect clock drifts, we can use the regular sequence of superframes as depicted in Fig. 1. The time is divided into pseudo slots, and the depth of a node gives deterministically the slot number used for its superframe. In particular, if a node uses the timeslot $k$ to transmit its superframe, its child will use the timeslot $k+1$.

We can note that the number of timeslots is bounded: we have a kind of modulo since a node with a large depth in the cluster-tree will re-use the timeslot of the PAN coordinator. We denote by $n_{\text {slot }}$ this bound. The cluster-tree should be constructed to minimize interferences among superframes (e.g. superframes of $A$ and $E$ in Fig. 1). Besides, we can note that $n_{\text {slot }}=\frac{B I}{S D}=2^{B O-S O}$ (in Fig. $1, n_{\text {slot }}=4$ ).

A node could sleep during one superframe if its address is not present in the pending address list of the corresponding beacon. However, for a sake of simplicity, we consider each node has at least one packet to transmit during each superframe. Since beacon intervals are practically long, this 


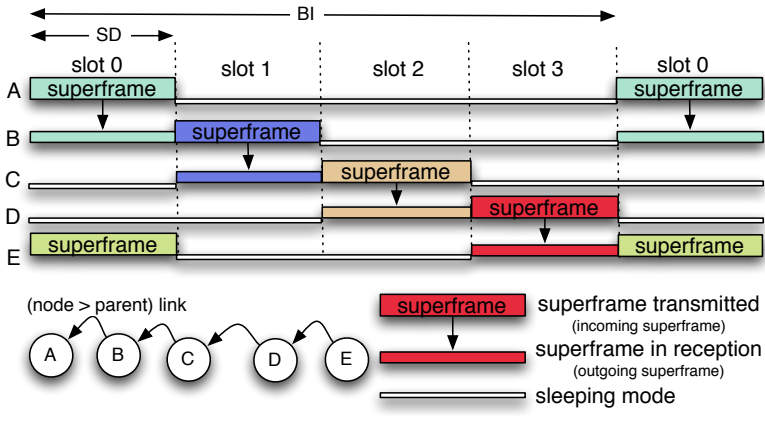

Fig. 1. The sequence of timeslots for the superframes $(\mathrm{BO}=\mathrm{SO}+2)$

assumption is realistic: a node would have an almost null traffic and could be neglected in the formulation if it participates to a so small number of superframes.

We differentiate the transmitting $\left(P_{t}\right)$, receiving $\left(P_{r}\right)$ and idle listening $\left(P_{i}\right)$ powers. Besides, we consider that a node in sleeping node doesn't consume energy (i.e. $P_{s}=0$ ). A nonnull energy in sleeping mode would only add an offset to the energy consumption for the other modes. Since we use a linear formulation, we would obtain the same results. For a sake of simplicity in the formulation, we assume a constant packet size. However, any formulation can be adopted, except the fact that the equations are consequently less concise.

We model the network with a graph $G=(V, E), V$ being the set of vertices and $E$ the set of edges. A conflict graph $G_{c}$ can be associated to the graph $G$ : each vertex of $G_{c}$ represents a radio link, and they are neighbor in $G_{c}$ if they interfere with each other. We denote by $N(u)$ the neighborhood of $u$ in $G$.

\section{CAPACITY AND ENERGY CONSUMPTION}

We will now describe how we can use Mixed Integer Linear Programming to define the network capacity and the energy consumption. This framework allows to evaluate formally a cluster-tree structure according to these two objectives.

This work constitutes a step toward understanding what are the limits of current cluster-trees, and how we can modify them accurately. However, the scope is larger: we conjecture that this MILP formulation can be adapted to other types of structures. For instance, the DAG structure of RPL can use the same formulation, coupling MAC and routing.

This letter constitutes a first step toward designing an efficient cluster-tree structure so that the superframe organization becomes efficient to distribute bandwidth.

a) Notations: Let define the MILP variables as follows:

- $f(u, v)$ is the normalized quantity of bandwidth consumed by the radio link from $u$ to $v$. It represents the fraction of time $u$ uses to transmit its packets to $v$;

- $f(u, v, k)$ denotes the traffic between $u$ and $v$ using the timeslot $k$;

- $f(u)$ is the quantity of data generated by the node $u$;

- $\operatorname{child}(u, v)$ is 1 if node $u$ is a child of $v$, else it is 0 ;

- $\operatorname{coord}(u)$ is 1 if node $u$ is a coordinator (non leaf node);

- $\operatorname{root}(u)$ is 1 if $u$ is the PAN coordinator, denoted by $P C$;

- superframe $(u, k)$ equals 1 iif the node $u$ uses the $k^{t h}$ superframe in the sequence;

- $\operatorname{slot}(u)$ equals $k$ if node $u$ uses the $k^{\text {th }}$ timeslot (iif superframe $(u, k)=1)$;
- $E_{u}$ represents the energy consumed by $u$. We denote by $T_{s d}$ the superframe duration.

- $\operatorname{act}(u, v, k)$ is 1 iif the link $(u, v)$ is active during slot $k$. b) Tree structure: first of all, a node can be associated to only one parent, and a coordinator is not a leaf:

$$
\begin{array}{r}
\forall u \in V, \quad \sum_{v \in N(u)} \operatorname{child}(u, v) \leq 1 \\
\forall u \in V, \quad \operatorname{coord}(u) \leq \sum_{v \in N(u)} \operatorname{child}(v, u)
\end{array}
$$

Only a link between one node and its parent is active:

$$
\forall(u, v) \in E, \quad f(u, v) \leq \operatorname{child}(u, v)
$$

c) Energy Consumption: a node consumes energy for its data reception/transmission. It receives a beacon only if it is not the PAN coordinator, and all non-leaf nodes send a beacon. It is idle listening during 1 superframe if its is a leaf, else during 2 superframes:

$$
\begin{gathered}
\forall u \in V, E_{u} \geq P_{t} \sum_{v \in N(u)} f(u, v)+P_{r} \sum_{v \in N(u)} f(v, u)+ \\
P_{r}(1-\operatorname{root}(u))+P_{t} \cdot \operatorname{coord}(u)+ \\
P_{i}\left(T_{s d}(1+\operatorname{coord}(u))-\left(\sum_{v \in N(u)} f(u, v)-\sum_{v \in N(u)} f(v, u)\right)\right)
\end{gathered}
$$

We can note that traffic $f(u, v)$ is a fraction of time, permitting to convert directly a power into energy.

We neglect here the fact that a node can turn-off its radio when it finished to transmit and receive its frames. We could take this phenomenon into account by computing the average time needed by a node to transmit and receive all its frames, according to the load of the other children.

d) Flow conservation: we have also the classical flow constraints: the traffic to the parent is equal to the traffic from children plus the traffic generated by the node itself.

$$
\forall u \in V-\{P C\}, \sum_{p \in N(u)} f(u, p)=\sum_{c \in N(u)} f(c, u)+f(u)
$$

The PAN coordinator is the sink for all the packets:

$$
\sum_{u \in N(P C)} f(u, P C)=\sum_{u \in V} f(u)
$$

e) superframe scheduling: A coordinator can coordinate only one superframe:

$$
\forall u \in V, \sum_{k \in\left[1, n_{\text {slot }}\right]} \text { superframe }(u, k) \leq \operatorname{coord}(u)
$$

the outgoing superframe is transmitted later than the incoming superframe:

$$
\begin{aligned}
& \forall(u, v) \in E, \operatorname{slot}(u) \geq \operatorname{slot}(v)+1 \\
& -(1-\operatorname{child}(u, v)) \times n_{\text {slot }} \\
& -\operatorname{superframe}\left(v, n_{\text {slot }}\right) \times n_{\text {slot }} \\
& \quad-(1-\operatorname{coord}(u)) \times n_{\text {slot }}
\end{aligned}
$$

The precedence between the superframes holds when we have a link in the tree (second line), if the parent $v$ has not the maximum slot (third line), and if $u$ is not a leaf (fourth line, because a leaf has no superframe). 


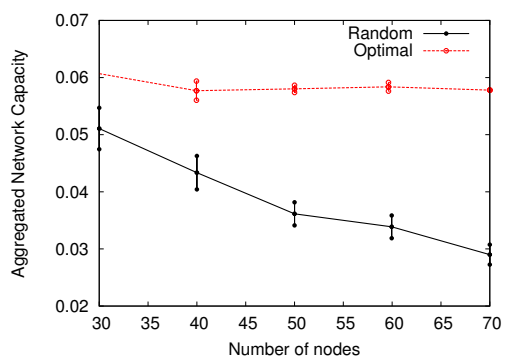

(a) Network capacity vs. nb. of nodes (avg. node degree of 8)

Fig. 2. Performances of the different cluster-tree algorithms

The superframes are immediately consecutive:

$$
\begin{aligned}
\forall(u, v) \in E, \operatorname{slot}(u)<\operatorname{slot}(v)+2 \\
+(1-\operatorname{child}(u, v)) \times n_{\text {slot }}
\end{aligned}
$$

Finally, if a node uses the largest slot $\left(n_{\text {slot }}\right)$, then its child has to use the slot 0 (we have a kind of modulo for timeslots):

$$
\begin{array}{r}
\forall(u, v) \in E, \quad \operatorname{slot}(u) \leq 2 n_{\text {slot }}-n_{\text {slot }} \times \operatorname{child}(u, v) \\
-n_{\text {slot }} \times \operatorname{superframe}\left(v, n_{\text {slot }}\right)
\end{array}
$$

f) Bandwidth sharing: nodes use the timeslot and superframe of their parent to exchange packets with it:

$\forall k \in\left[0 . . n_{\text {slot }}-1\right], \forall(u, v) \in E, f(u, v, k) \leq \operatorname{superframe}(v, k)$

$\forall(u, v) \in E, \forall k \in\left[0 . . n_{\text {slot }}-1\right], f(u, v, k) \leq \operatorname{act}(u, v, k)$

$$
\forall(u, v) \in E, \quad \sum_{k} f(u, v, k)=f(u, v)
$$

All the interfering links that share the same slot must share the radio bandwidth (equal to 1 unit, divided by the number of slots) minus the bandwidth wasted by collisions as described in eq. 1 :

$$
\begin{aligned}
& \forall k \in\left[0 . . n_{\text {slot }}-1\right], \forall c \in \mathcal{C}, \sum_{(u, v) \in c} f(u, v, k) \leq \\
& \frac{1}{\left|n_{\text {slot }}\right|}\left(1-0.018675 \sum \operatorname{act}(u, v, k)-0.0104\right)
\end{aligned}
$$

with $\mathcal{C}$ being all the cliques in the conflict graph $G_{c}$ (i.e. radio links that interfere with each other).

g) Objective: we chose to maximize the network fair capacity:

$$
\text { Obj : } \max (\min (f(u)))
$$

\section{Performance Evaluation}

We generated random topologies of nodes following the well known Unit-Disk-Graph model. The nodes are distributed randomly in a circular area, the PAN coordinator being placed in the center. All disconnected network's instances are simply discarded. The formulation introduced in the precedent section entirely describes the bandwidth sharing and the superframe organization in IEEE 802.15.4. Thus, we just have to feed our MILP solver (CPLEX version 12.0) with the linear constraints to obtain the variable's values. To study the behavior of a particular algorithm, we just have to fix the variables corresponding to the tree organization (the child $(u, v)$ ). We plotted the $95 \%$ confidence intervals. Because of lack of space, we only plotted results for 4 timeslots, but we verified that different values lead to similar results. For the same reason,

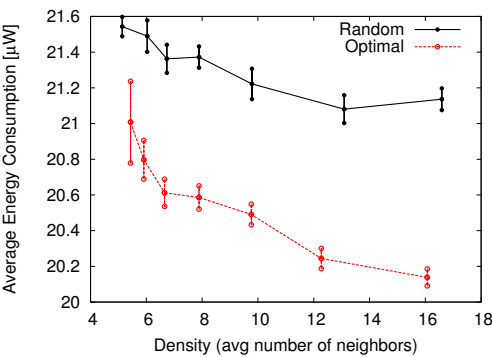

(c) Energy consumption versus node degree (50 nodes)

we present only the results for the network capacity and the energy consumption.

We first vary the number of nodes. We compared the optimal cluster-tree with the random strategy (a node chooses the first available parent transmitting a beacon). We note that the random strategy does not lead to the best network capacity (Fig. 2(a)). The optimal cluster-tree achieves a much better throughput, and it is scalable: its performances are not impacted by the network cardinality.

We also measured the influence of the average node degree (Fig. 2(b)). A dense WSN permits to construct different cluster-trees. The optimal strategy leads to better results: less collisions may occur since the nodes own to different superframes. On the contrary, the random strategy does not achieve to exploit this property. However, the optimal strategy keeps on presenting a better capacity. We can also remark that, surprisingly, a cluster-tree which presents the best capacity also optimizes the energy consumption (Fig. 2(c)). In other words, an association to the first available parent is simple to implement but gives poor throughput and energy consumption performances.

\section{Conclusion \& Perspectives}

We developed here a MILP formulation providing a formal framework to evaluate a cluster-topology structure. While we focus here on maximizing the network capacity, we can easily develop other objectives such as minimizing the energy consumption. Besides, this formulation constructs an optimal cluster-tree concerning this objective. The performance evaluation demonstrated that an opportunistic approach (i.e. a node associates to any already associated neighbor) is inefficient and an efficient cluster-tree strategy has still to be proposed. We plan to study now the characteristics of the optimal clustertree topology and to propose a distributed algorithm leading to good performances concerning these criteria.

\section{REFERENCES}

[1] IEEE 802.15.4-2006 standard, http://www.ieee802.org/15/pub/TG4.html.

[2] F. Cuomo et al. Topology formation in IEEE 802.15.4: Cluster-tree characterization. In PerCom. IEEE, 2008.

[3] F.J. Claudios et al. Performance study of reconfiguration algorithms in cluster-tree topologies for wireless sensor networks. In PIMRC, 2007.

[4] A. Koubâa et al. TDBS: a time division beacon scheduling mechanism for zigbee cluster-tree wireless sensor networks. Real-Time Systems, 40(3):321-354, December 2008.

[5] S. Pollin et al. Performance analysis of slotted carrier sense ieee 802.15.4 medium access layer. IEEE Transactions on Wireless Communications, 7(9):3359-3371, September 2008. 